\title{
Low-dimensional factors of superelliptic Jacobians
}

\author{
Thomas Occhipinti · Douglas Ulmer
}

Received: 30 September 2014 / Revised: 20 October 2014 / Accepted: 25 October 2014 /

Published online: 30 December 2014

(C) Springer International Publishing AG 2014

\begin{abstract}
Given a polynomial $f \in \mathbb{C}[x]$, we consider the family of superelliptic curves $y^{d}=f(x)$ and their Jacobians $J_{d}$ for varying integers $d$. We show that for any integer $g$ the number of abelian varieties up to isogeny of dimension $\leq \mathrm{g}$ which appear in any $J_{d}$ is finite and their multiplicities are bounded.
\end{abstract}

Keywords Jacobian $\cdot$ Superelliptic curve $\cdot$ Abelian variety

Mathematics Subject Classification $\quad 14 \mathrm{H} 40 \cdot 14 \mathrm{H} 45 \cdot 14 \mathrm{~K} 12$

\section{Introduction}

Given a family of abelian varieties, it is often interesting to ask about the existence and multiplicity of small-dimensional abelian subvarieties. For example, in [3], Ekedahl and Serre ask several questions about curves over $\mathbb{C}$ whose Jacobian is isogenous to a power of an elliptic curve.

Related questions about Fermat Jacobians are discussed in [7]. Let $F_{d}$ denote the Fermat curve of degree $d$ over a field of characteristic zero. In [7, Theorem 3.2] it is proven that for every $g>0$, only finitely many abelian varieties up to isogeny of dimension $\leq \mathrm{g}$ appear as subvarieties of the Jacobian of $F_{d}$ for any $d$. The goal of this note is to generalize that result to a large class of superelliptic curves. Our results apply

\footnotetext{
T. Occhipinti

Department of Mathematics, Carleton College, One North College Street, Northfield, MN 55057, USA e-mail: tocchipinti@carleton.edu

D. Ulmer $(\bowtie)$

School of Mathematics, Georgia Institute of Technology, 686 Cherry Street, Atlanta, GA 30332, USA

e-mail: douglas.ulmer@math.gatech.edu
} 
over any field of characteristic zero, but for simplicity of language we work over the complex numbers $\mathbb{C}$.

Fix a polynomial $f \in \mathbb{C}[x]$ of degree $n>0$ and let $e$ be the largest positive integer such that $f=f_{0}^{e}$ where $f_{0}$ is a polynomial. If $d$ is a positive integer which is relatively prime to $e$, then the polynomial $y^{d}-f(x)$ is irreducible. We may thus define $C_{f, d}$ to be the smooth, projective curve over $\mathbb{C}$ associated to the affine plane curve

$$
y^{d}-f(x)=0
$$

If $f_{0}$ is linear, then the genus of $C_{f, d}$ is zero for all $d$ prime to $e$. On the other hand, if $\operatorname{deg} f_{0}>1$, then the genus of $C_{f, d}$ tends to infinity with $d$.

Here is our main result:

Theorem 1.1 For a fixed polynomial $f$ as above and a fixed positive integer $g$, only finitely many abelian varieties up to isogeny of dimension $\leq g$ appear as subvarieties of the Jacobians of $C_{f, d}$ as $d$ varies over all positive integers relatively prime to $e$.

This result can be used to give more examples of isotrivial elliptic curves (and abelian varieties) over $\mathbb{C}(t)$ which have bounded Mordell-Weil ranks in the tower of extensions $\mathbb{C}\left(t^{1 / d}\right)$, along the lines of the discussion in [7, Section 4]. We leave the details as an exercise for the reader.

We fix $f$ and write $J=J_{d}$ for the Jacobian of $C_{f, d}$. It will be convenient to reformulate the theorem in terms of the "new part" of $J$. Note that if $d^{\prime}$ divides $d$, then there is a surjective morphism $C_{f, d} \rightarrow C_{f, d^{\prime}}$ which induces a homomorphism of Jacobians $J_{d^{\prime}} \rightarrow J_{d}$. We define $J^{\text {new }}=J_{d}^{\text {new }}$ to be the quotient of $J_{d}$ by the sum of the images of these morphisms for all proper divisors $d^{\prime}$ of $d$. It is not hard to show that $J_{d}$ is isogenous to the product of $J_{d^{\prime}}^{\text {new }}$ with $d^{\prime}$ ranging over all divisors of $d$. Theorem 1.1 is thus implied by the following result:

Theorem 1.2 For all integers $n>0$ and $g>0$, there is a constant $d_{0}$ depending only on $n$ and $g$ with the following property: if $f$ is a polynomial of degree $n$ and $e$ is the largest positive integer such that $f=f_{0}^{e}$, and if $d$ is relatively prime to e and $>d_{0}$, then $J_{d}^{\text {new }}$ has no abelian subvarieties of dimension $\leq g$.

Note that Theorem 1.2 is stronger than Theorem 1.1 because it shows that the dependence on $f$ is only through its degree.

The proof of Theorem 1.2 will be given in Sects. 2 through 6. At the end of Sect. 6 we discuss what we know or expect in other contexts, such as when $f$ is a rational function or when the ground field has positive characteristic.

\section{The complex representation}

We keep the notations and definitions of the introduction. The curve $C$ is a Galois cover of $\mathbb{P}^{1}$ with Galois group the $d$ th roots of unity $\mu_{d}$. In terms of equation (1), $\mu_{d}$ acts via multiplication on the coordinate $y$. We will study the action of $\zeta$ on the regular 1 -forms of $C$, or equivalently on the regular 1 -forms of $J$. This is sometimes called the complex representation of $J$. 
Fix once and for all a primitive $d$ th root of unity $\zeta$. We have a direct sum decomposition

$$
H^{0}\left(J, \Omega^{1}\right)=H^{0}\left(C, \Omega^{1}\right)=\bigoplus_{j=0}^{d-1} V_{j}
$$

with $V_{j}$ the subspace where $\zeta$ acts by multiplication by $\zeta^{j}$. It will be convenient to view the subscripts $j$ as lying in $\mathbb{Z} / d \mathbb{Z}$.

Note that since $C_{f, d^{\prime}}$ is the quotient of $C_{f, d}$ by the subgroup of $\mu_{d}$ generated by $\zeta^{d^{\prime}}$, we may identify $H^{0}\left(C_{f, d^{\prime}}, \Omega^{1}\right)$ with the subspace

$$
\bigoplus_{j=0}^{d^{\prime}-1} V_{\left(d / d^{\prime}\right) j}
$$

It follows that we have

$$
H^{0}\left(J^{\text {new }}, \Omega^{1}\right) \cong \bigoplus_{j \in(\mathbb{Z} / d \mathbb{Z})^{\times}} V_{j}
$$

In particular, the roots of the characteristic polynomial of $\zeta$ on $H^{0}\left(J^{\text {new }}, \Omega^{1}\right)$ are primitive $d$ th roots of unity.

A crucial point in our analysis is that the dimensions of $V_{j}$ are not evenly distributed. More precisely:

Proposition 2.1 If $j \in(\mathbb{Z} / d \mathbb{Z})^{\times}$has least positive residue $<d / n=d / \operatorname{deg} f$, then $V_{j}=0$.

Proof A standard calculation gives a basis of 1-forms on $C$ consisting of forms of the shape $g(x) d x / y^{k}$, and the dimension of $V_{j}$ is the number of basis elements with $k \equiv-j(\bmod d)$. See [1, Section 6] for a detailed account. The proposition is an immediate consequence of the dimension formula [1, Theorem 6.7].

For the convenience of the reader, we restate [1, Theorem 6.7] using our notation. For this, write

$$
f_{0}=c \prod_{i=1}^{m}\left(x-\lambda_{i}\right)^{e_{i}}
$$

Then the formula in question says that if $\operatorname{gcd}(j, d)=1$ and $d$ does not divide any of $e e_{1}, \ldots, e e_{m}$ nor $n$, then

$$
\operatorname{dim} V_{j}=-\left\langle\frac{j n}{d}\right\rangle+\sum_{i=1}^{m}\left\langle\frac{j e e_{i}}{d}\right\rangle .
$$


Here $\langle r\rangle$ denotes the fractional part of a real number $r$. It follows that if $d>n$ and $j<d /\left(e \max \left\{e_{i}\right\}\right) \leq d / n$, then $\operatorname{dim} V_{j}=0$, and this is exactly the assertion of the proposition.

\section{Consequences of a small factor}

In this section we show that if $J^{\text {new }}$ has an abelian subvariety of small dimension, then the characteristic polynomial of $\zeta$ on $H^{0}\left(J^{\text {new }}, \Omega^{1}\right)$ has a factor with coefficients in a number field of small degree. More precisely:

Proposition 3.1 Suppose that $J^{\text {new }}$ has an abelian subvariety of positive dimension $\leq g$. Then there exists a number field $K \subset \mathbb{C}$ of degree $\leq 2 \mathrm{~g}$ and a polynomial $P$ of positive degree with coefficients in $K$ such that $P$ divides the characteristic polynomial on $\zeta$ on $H^{0}\left(J^{\text {new }}, \Omega^{1}\right)$.

Note that we may take the polynomial $P$ to be irreducible over $K$. Note also that the roots of $P$ are primitive $d$ th roots of unity.

Proof Suppose that $A \subset J^{\text {new }}$ is an abelian subvariety of positive dimension $\leq g$. We may assume that $A$ is simple. Let $a>0$ be the integer such that $J^{\text {new }}$ is isogenous to $A^{a} \oplus B$ where $B$ is an abelian variety with no factors isogenous to $A$. Fixing an isogeny yields an identification

$$
H^{0}\left(J^{\text {new }}, \Omega^{1}\right) \cong H^{0}\left(A^{a}, \Omega^{1}\right) \oplus H^{0}\left(B, \Omega^{1}\right)
$$

Since $A$ and $B$ have no isogeny factors in common, the action of $\zeta$ preserves the subspace $H^{0}\left(A^{a}, \Omega^{1}\right)$. Our claim will follow from an inspection of the characteristic polynomial of $\zeta$ on $H^{0}\left(A^{a}, \Omega^{1}\right)$.

Let $D$ be the rational endomorphism algebra of $A: D=\operatorname{End}(A) \otimes \mathbb{Q}$. Then it is well known [4, Section 19] that $D$ is a simple algebra whose center $K$ is a number field of degree $\leq 2 g$. Let $[D: K]=f^{2}$. The endomorphism algebra of $A^{a}$ is $M_{a}(D)$, and the irreducible complex representations of this algebra are indexed by the embeddings of $K$ into $\mathbb{C}$. Given an embedding, the corresponding representation is the composition

$$
M_{a}(D) \hookrightarrow M_{a}(D) \otimes_{K} \mathbb{C} \cong M_{a f}(\mathbb{C})
$$

(where the embedding is used to form the tensor product) acting by left multiplication on $\mathbb{C}^{a f}$. If $\delta \in M_{a}(D)$, the eigenvalues of the action of left multiplication by $\delta$ on the $K$-vector space $M_{a}(D)$ are the eigenvalues of the corresponding element of $M_{a f}(\mathbb{C})$ each repeated af times. It follows that the characteristic polynomial of the element $\delta$ in either representation has coefficients in $K$. Therefore, we may take $P$ to be the characteristic polynomial of $\zeta$ on an irreducible constituent of $H^{0}\left(A^{a}, \Omega^{1}\right)$ viewed as a representation of $M_{a}(D)$. 


\section{Galois theory}

Next we consider the distribution of roots of a polynomial with coefficients in a small field and with a root which is a root of unity. More precisely:

Lemma 4.1 Let $P$ be an irreducible polynomial with coefficients in a number field $K$ of degree $\leq 2 g$ over $\mathbb{Q}$. Suppose that $P$ has primitive dth roots of unity as roots. Then there exists a subgroup $H \subset(\mathbb{Z} / d \mathbb{Z})^{\times}$of index $\leq 2 \mathrm{~g}$ such that the roots of $P$ are of the form $\zeta^{a b}$ for a fixed $a \in(\mathbb{Z} / d \mathbb{Z})^{\times}$and $b \in H$.

Proof This is a simple exercise in Galois theory: Since the roots of $P$ lie in $\mathbb{Q}\left(\mu_{d}\right)$, we may assume that $K$ is a subfield of $\mathbb{Q}\left(\mu_{d}\right)$. The splitting field of $P$ is $\mathbb{Q}\left(\mu_{d}\right)$, so the roots of $P$ are some fixed primitive $d$ th root $\zeta^{a}$ and its conjugates under $H=$ $\operatorname{Gal}\left(\mathbb{Q}\left(\mu_{d}\right) / K\right)$.

\section{Equidistribution}

The last step in the proof of the main theorem is to note that subgroups of $(\mathbb{Z} / d \mathbb{Z})^{\times}$ with small index are in a certain sense equidistributed. More precisely:

Proposition 5.1 Given positive integers $n$ and $g$, there exists an integer $d_{0}$ depending only on $n$ and $g$ such that if $d>d_{0}$ and $H \subset(\mathbb{Z} / d \mathbb{Z})^{\times}$is a subgroup of index $2 g$, then every coset of $H$ has an element whose least residue lies in the interval $(0, d / n)$.

Proof The proposition follows from the following more precise statement: If for each $d$ we have a subgroup $H_{d} \subset G_{d}=(\mathbb{Z} / d \mathbb{Z})^{\times}$of index $\leq 2 g$, then as $d \rightarrow \infty$ the subsets $\left\{e^{2 \pi i b / d}: b \in H_{d}\right\}$ become equidistributed with respect to Haar measure on the circle.

This equidistribution statement follows easily from the Weyl equidistribution criterion (see [9] or [5, 4.2]) and standard estimates for Gauss sums (see [2, 2.1.6]). What is to be shown is that for all integers $a>0$, the quantity

$$
\frac{1}{\left|H_{d}\right|} \sum_{b \in H_{d}} \zeta_{d}^{a b}
$$

tends to zero as $d \rightarrow \infty$. We have

$$
\begin{aligned}
\left|\frac{1}{\left|H_{d}\right|} \sum_{b \in H_{d}} \zeta_{d}^{a b}\right| & =\frac{1}{\left|H_{d}\right|}\left|\sum_{b \in G_{d}}\left(\frac{1}{\left[G_{d}: H_{d}\right]} \sum_{\chi \in \widehat{G_{d} / H_{d}}} \chi(b)\right) \zeta_{d}^{a b}\right| \\
& \leq \frac{1}{\phi(d)} \sum_{\chi \in \widehat{G_{d} / H_{d}}}\left|\sum_{b \in G_{d}} \chi(b) \zeta_{d}^{a b}\right| \leq \frac{2 g}{\phi(d)} \sqrt{a d}
\end{aligned}
$$

where the last step uses standard estimates for Gauss sums. The last displayed quantity goes to zero as $d \rightarrow \infty$. This establishes the desired equidistribution. It follows that for large $d$, each coset of $H_{d}$ has an element whose least residue lies in $(0, d / n)$, as desired. 


\section{Conclusion and complements}

\subsection{Proof of Theorem 1.2}

Fix integers $n$ and $g$ and let $d_{0}$ be the integer associated to $n$ and $g$ by Proposition 5.1. If necessary, we may increase $d_{0}$ so that $d_{0}>n$. Fix a polynomial $f$ of degree $n$, form the curves $C_{f, d}$ for varying $d$, and consider the new parts of their Jacobians $J_{d}^{\text {new }}$.

Suppose that $A$ is an abelian variety of dimension $\leq g$ which appears in $J_{d}^{\text {new }}$ for some $d>d_{0}$. By Proposition 3.1, the characteristic polynomial of $\zeta$ on $H^{0}\left(J_{d}^{\text {new }}, \Omega^{1}\right)$ has a factor $P$ with coefficients in a number field $K$ of degree $\leq 2 g$ over $\mathbb{Q}$. By Lemma 4.1 and Proposition 5.1, $P$ has a root of the form $\zeta^{b}$ with $0<b<d / n$ and $\operatorname{gcd}(b, d)=1$. This implies that $V_{b} \neq 0$, in contradiction to Proposition 2.1. Therefore, no such $A$ exists, and this proves Theorem 1.2.

\subsection{Effectivity}

It would be interesting to make the bound $d_{0}$ effective. For $g=1$ and $n=2$, previous unpublished work of the first author shows that Theorem 1.2 holds with $d_{0}=10^{11}$. It can then be shown computationally that in fact $d_{0}=24$ is sufficient. At least insofar as Proposition 5.1 is concerned, this cannot be improved further because $\{1,5,7,11\}$ is an index 2 subgroup of $(\mathbb{Z} / 24 \mathbb{Z})^{\times}$.

We also note that if $f$ has degree $\geq 3$ and no repeated roots, and if $d$ is relatively prime to $n$ and sufficiently large ( $d>24$ suffices), then by [10, Corollary 1.11], $J_{d}$ is not isogenous to a product of elliptic curves.

\subsection{Characteristic $p$}

We note that the restriction to characteristic zero is essential: Theorem 1.1 and similar results are spectacularly false in characteristic $p$. Indeed, Tate and Shafarevich [6] showed that the Jacobian of the Fermat curve of degree $p^{f}+1$ is isogenous to a product of elliptic curves. More generally, a construction in [8, Section 10] yields many superelliptic curves whose Jacobians have an elliptic curve as factor with large multiplicity in the new part of their Jacobians.

All these examples involve supersingular elliptic curves. Comparing with [7, Theorem 3.3], one might hope that introducing restrictions like ordinarity or positive $p$-rank on small dimensional factors $A$ might yield a true variant of Theorem 1.1.

\subsection{Rational functions}

Finally, it would be natural to allow $f$ to be a rational function, not just a polynomial, in Theorem 1.1. We expect the result to continue to hold in this generality, but our proof breaks down because allowing poles in $f$ makes the "ill-distribution" result of Proposition 2.1 false. For example, if $f$ is a rational function with distinct zeroes and poles, then the dimensions of all of the $V_{j}$ with $j \in(\mathbb{Z} / d \mathbb{Z})^{\times}$are the same. Of course, 
if $f$ is a rational function, the curve defined by $y^{d}=f(x)$ can also be defined (using a suitable change of variables) by an equation $y^{d}=h(x)$ with $h$ a polynomial, but now $h$ will have large degree and this also spoils our argument.

Results in the opposite direction, namely examples where a given small-dimensional abelian variety appears in $J_{d}^{\text {new }}$ for infinitely many $d$, would also very interesting due to their relevance for ranks of abelian varieties and questions like those of Ekedahl and Serre.

Acknowledgments We thank the anonymous referee for a careful reading of the paper and for several useful suggestions.

\section{References}

1. Archinard, N.: Hypergeometric abelian varieties. Can. J. Math. 55(5), 897-932 (2003)

2. Cohen, H.: Number Theory, I. Tools and Diophantine Equations. Graduate Texts in Mathematics, vol. 239. Springer, New York (2007)

3. Ekedahl, T., Serre, J.-P.: Exemples de courbes algébriques à jacobienne complètement décomposable. C. R. Acad. Sci. Paris Sér. I Math. 317(5), 509-513 (1993)

4. Mumford, D.: Abelian Varieties. Tata Institute of Fundamental Research Studies in Mathematics, vol. 5. Hindustan Book, New Delhi (2008)

5. Stein, E.M., Shakarchi, R.: Fourier Analysis. Princeton Lectures in Analysis, vol. 1. Princeton University Press, Princeton (2003)

6. Tate, J.T., Shafarevich, I.R.: The rank of elliptic curves. Sov. Math. Dokl. 8, 917-920 (1967)

7. Ulmer, D.: Jacobi sums, Fermat Jacobians, and ranks of abelian varieties over towers of function fields. Math. Res. Lett. 14(3), 453-467 (2007)

8. Ulmer, D.: $L$-functions with large analytic rank and abelian varieties with large algebraic rank over function fields. Invent. Math. 167(2), 379-408 (2007)

9. Weyl, H.: Über die Gleichverteilung von Zahlen mod. Eins. Math. Ann. 77(3), 313-352 (1916)

10. Xue, J., Zarhin, Yu.G.: Centers of Hodge groups of superelliptic Jacobians. Transform. Groups 15(2), 449-482 (2010) 